TRANSACTIONS OF THE

AMERICAN MATHEMATICAL SOCIETY

Volume 361, Number 1, January 2009, Pages 161-176

S 0002-9947(08)04420-6

Article electronically published on August 13, 2008

\title{
GEODESIC CURRENTS AND LENGTH COMPACTNESS FOR AUTOMORPHISMS OF FREE GROUPS
}

\author{
STEFANO FRANCAVIGLIA
}

\begin{abstract}
We prove a compactness theorem for automorphisms of free groups. Namely, we show that the set of automorphisms keeping the length of the uniform current bounded is compact (up to conjugation). This implies that the spectrum of the length of the images of the uniform current is discrete, proving a conjecture of I. Kapovich.
\end{abstract}

\section{INTRODUCTION}

The aim of this paper is to investigate the action of the automorphisms of a free group $F$ on the Cayley graph of $F$. In particular, we are interested in understanding how automorphisms can stretch geodesics. One can define the length of an automorphism as the generic stretching factor (see KKS05]) which is, roughly speaking, the average of the stretching ratios, taken over all geodesics (Definition 3.7). Intuitively, for $\Phi \in \operatorname{Aut}(F)$, one can think of its length as the limit, as $n$ tends to infinity, of $\left\|\Phi\left(w_{n}\right)\right\| / n$, where $w_{n}$ is a "random" cyclically reduced word in $F$ of length $n$, and $\|\cdot\|$ denotes the cyclically reduced length.

The length function on $\operatorname{Aut}(F)$ is invariant under conjugation, so it descends to a length function on $\operatorname{Out}(F)$. Our main result is the following:

Theorem 1.1 (Length compactness theorem). Let $\Phi_{n}$ be a sequence of automorphisms of $F$. Then, up to passing to subsequences, there exists a sequence $v_{n} \in F$ such that the automorphisms $\Psi_{n}$ defined by $x \mapsto v_{n} \Phi(x) v_{n}^{-1}$ satisfy one (and only one) of the following:

- $\Psi_{n}$ converges to an automorphism $\Phi$ (that is, it has a constant subsequence $\left.\Psi_{n_{i}}=\Phi\right)$.

- $L\left(\Phi_{n}\right)$ goes to $\infty$.

A reformulation of this theorem is the following:

The set of automorphisms of bounded length is compact up to conjugation. That is, for any $M \in \mathbb{R}$, the set $\{[\Phi] \in \operatorname{Out}(F): L(\Phi)<M\}$ is finite.

Equivalently: For a sequence of automorphisms $\Phi_{n}$, if there is a word $w$ such that the cyclically reduced length of $\Phi_{n}(w)$ goes to $\infty$, then $L\left(\Phi_{n}\right) \rightarrow \infty$.

All the work pivots on the fact that the Cayley graph of $F$ is a hyperbolic object. Therefore, the boundary at infinity $\partial F$ of $F$ is well defined, and encodes enough information about the dynamic of the action of $\operatorname{Aut}(F)$. The main idea is

Received by the editors May 22, 2006 and, in revised form, October 25, 2006.

2000 Mathematics Subject Classification. Primary 20F65.

Key words and phrases. Automorphisms, free groups, geodesic currents.

The author was supported by a Marie Curie Intra European Fellowship.

(c) 2008 American Mathematical Society Reverts to public domain 28 years from publication 
that length controls attractors: if $\Phi_{n}$ is a sequence of automorphisms of bounded length, then, up to conjugation, there are no attractors for the action of $\Phi_{n}$ on $\partial F$. Using this fact we prove that the sequence $\Phi_{n}$ keeps the cyclically reduced length of any element of $F$ bounded, and this implies that $\Phi_{n}$ has a constant subsequence.

As a consequence of Theorem 1.1, we get the following result.

Corollary 1.2. The spectrum of the length function is discrete. That is, the set

$$
\{L(\Phi): \Phi \in \operatorname{Aut}(F)\}
$$

is a discrete subset of $\mathbb{R}$.

We remark that, while the length of an automorphism depends on the free basis chosen for the Cayley graph, the spectrum of the length function is intrinsic (it depends in fact on the current we use to define it, which in our case is the uniform current).

Corollary 1.2 was conjectured to be true by I. Kapovich, inspired by computational evidence and partial results. For example, in KKS05 V. Kaimanovich, I. Kapovich and P. Shupp proved (among other results) that an automorphism of length one must be simple (see below) and estimated the "first gap" of the length function.

A consequence of Corollary 1.2 is that one can use inductive arguments on the length. For example, we get the following result, which can be viewed as an Ideal Whitehead Algorithm (see Kap06a, Conjecture 5.3]). Recall that, given a free basis $\Sigma$ of $F$, an automorphism $\tau$ is simple if it is either a permutation of $\Sigma$ or an inner automorphism, while it is a Whitehead automorphism of the second kind if there is $a \in \Sigma$ such that $\tau(x) \in\left\{x, x a, a^{-1} x, a^{-1} x a\right\}$ for all $x \in \Sigma$ (see for example [LS77] for more details).

Theorem 1.3. Let $\Phi \in A u t(F)$ be a non-simple automorphism. Then there exists a factorisation

$$
\Phi=\tau_{n} \tau_{n-1} \cdots \tau_{1} \sigma
$$

where $n \geq 1$, the automorphism $\sigma$ is simple, each $\tau_{i}$ is a Whitehead automorphism of the second kind, and

$$
L\left(\tau_{i-1} \cdots \sigma\right)<L\left(\tau_{i} \tau_{i-1} \cdots \sigma\right), \quad i=1, \cdots, n-1 .
$$

Let us say a few words about Theorem 1.3. The automorphism problem for a free group $F$ asks, given two arbitrary elements $u, v \in F$, whether there exists $\Phi \in \operatorname{Aut}(F)$ such that $\Phi(u)=v$. In Whi36] Whitehead gave an algorithm solving that problem. The first part of the algorithm is to maximally reduce the lengths of $u$ and $v$ via Whitehead automorphisms. Then, given two minimal elements one proves that they are in the same $\operatorname{Aut}(F)$-orbit if and only if they are related via a sequence of minimal elements, each one obtained from the preceding via a Whitehead automorphism. Roughly speaking, Theorem 1.3 is an averaged version of the first part of the Whitehead algorithm. We refer the reader to Kap06a for a more detailed discussion on the matter. We only notice that, as our proof is "typically hyperbolic," one may expect that it could be adapted to a more general setting, like that of hyperbolic groups, for which the automorphism problem is still not completely solved.

The main tool we use is the theory of geodesic currents. These are locally finite $F$-invariant Borel measures on the space of geodesics lines in the Cayley graph of 
F. Geodesic currents where introduced by F. Bonahon [Bon86 in the setting of hyperbolic manifolds, and turned out to be very useful in group theory (see for example [Mar95, Kap06b, Kap06a).

We also consider measures on the space of geodesic rays (that is, half geodesics) in the Cayley graph of $F$. This is the space of frequency measures (see also Kap05).

Such spaces are in fact homeomorphic, but each one has peculiar characteristics that are well-adapted to different situations, and we will jump from one to the other depending on the calculations needed. Roughly speaking, the action of $\operatorname{Aut}(F)$ on currents is "more natural", while frequency measures are "more compact".

As mentioned earlier, the length function on $\operatorname{Aut}(F)$ depends on the choice of a current. We use the uniform current, which is the analogous of the Liouville current for the geodesic flow of a surface, but, ça va sans dire, length compactness should hold for many other currents. In the last section we discuss some generalisations of Theorem 1.1 in such a direction.

Remark 1.4. The space of automorphisms of a free group is discrete. Thus, compactness is equivalent to finiteness, and to say that a sequence converges is equivalent to saying that it is finite (and hence eventually constant). Nevertheless, we prefer to speak about compactness and convergence because we think that this is closer to the spirit of the paper, in which we used "more hyperbolicity than discreteness". (Even if discreteness is necessary, as, for example, in Corollary 1.2.)

\section{Notation}

For the remainder of the paper, we fix the following notation:

- $F$ is a free group of rank $k$, with a fixed free basis $\Sigma$. We set $A=\Sigma \cup$ $\Sigma^{-1}$. Any element of $F$ corresponds to a unique freely reduced word in the alphabet $A$, that is, a word not containing sub-words of the form $a a^{-1}$ with $a \in A$. We identify $F$ with the set of freely reduced words. We denote by 1 the neutral element of $F$ (the empty word). A word $w$ is cyclically reduced if all the cyclic permutations of $w$ are freely reduced. For $v$ a freely reduced word, $|v|$ denotes its length, and $\|v\|$ denotes its cyclically reduced length, that is, the length of the cyclically reduced word obtained by cyclically reducing $v$.

- The Cayley graph of $F$ corresponding to $A$ will shortly be denoted simply by a Cayley graph. We denote by 1 the base point of the Cayley graph corresponding to $1 \in F$.

- $\partial F$ is the boundary at infinity of $F$, identified with the set of geodesic rays in the Cayley graph, that is, freely reduced, right-infinite words in the alphabet $A$. The boundary $\partial F$ is endowed with the Cantor-set topology. Namely, for each $v \in F$ we denote by $\operatorname{Cyl}(v)$ the set of rays having $v$ as an initial segment. We set $\operatorname{Cyl}(1)=\partial F$. Then, a basis for the topology of $\partial F$ is given by the sets $\{\mathrm{Cyl}(v): v \in F\}$.

- $\partial^{2} F$ is the set $\left\{(x, y) \in(\partial F)^{2}: x \neq y\right\}$. We identify $\partial^{2} F$ with the set of oriented bi-infinite geodesics in the Cayley graph. We define the base-ball $B$ of $\partial^{2} F$ as the set of geodesics passing through 1 .

- For any $x \neq y \in F$, the cylinder $\operatorname{Cyl}([x, y])$ is defined as the subset of $\partial^{2} F$ of geodesics passing through the oriented segment joining $x$ and $y$ in the Cayley graph (with the correct orientation). We set $\operatorname{Cyl}([1,1])=B$. 
- We denote by $T: \partial F \rightarrow \partial F$ the shift operator deleting the first letter of a ray. It turns out that $T$ is a continuous map.

- Given a topological space $M$, we identify the space of Borel measures on $M$ with the dual of $\mathcal{C}_{0}(M)$ (the space of continuous functions on $M$ with compact support) endowed with the weak-* topology. Namely, measures $\mu_{i}$ converge to $\mu$ if and only if $\int \varphi d \mu_{i} \rightarrow \int \varphi d \mu$ for all $\varphi \in \mathcal{C}_{0}(M)$. If $\mu$ is a Borel measure on $M, N$ is a topological space, and $f: M \rightarrow N$ is a measurable, proper map, we denote by $f_{*} \mu$ the push-forward of $\mu$, that is, the measure on $N$ such that $\int_{N} \varphi d\left(f_{*} \mu\right)=\int_{M} \varphi \circ f d \mu$ for all $\varphi \in \mathcal{C}_{0}(N)$ (see for example the first chapters of [Fed69] or [AFP00] for good introductions to geometric measure theory).

\section{DEFINITIONS AND PRELIMINARY FACTS}

In this section, we define the space of geodesic currents and of frequency measures, and we show that such spaces are homeomorphic. We introduce the length of a current, which is the analog of the length of a cyclically reduced word. We define the uniform frequency measure and the uniform current, which we use to define the length of automorphisms.

First of all, in order to describe the action of $\operatorname{Aut}(F)$ on currents, we need the following classical result, whose proof can be found in Coo87.

Theorem 3.1. Let $\Phi$ be an automorphism of $F$. Then it extends to a homeomorphism of $\partial F$ (which we still denote by $\Phi)$.

Since $\Phi$ is a homeomorphism of $\partial F$, the map $\Phi \times \Phi$ is continuous and proper on $\partial^{2} F$. It follows that any automorphism $\Phi$ acts on the space of Borel measures on $\partial^{2} F$ via $(\Phi \times \Phi)_{*}$. The inclusion of $F$ in $\operatorname{Aut}(F)$ given by inner automorphisms induces an action of $F$ on the space of Borel measures on $\partial^{2} F$. By abuse of notation, if $\eta$ is a Borel measure, we will denote by $\Phi \eta$ the measure $(\Phi \times \Phi)_{*} \eta$. We can now give the definition of currents and frequency measures. Our definitions are a little different from those introduced in Kap06b, Kap05, as we do not require measures to be normalised to probability measures. This is because the quantities we are interested in (lengths of automorphisms) depend on the total mass of the measures with which we work.

Definition 3.2 (Geodesic currents and their lengths). The space of geodesic currents is the space of locally finite (i.e. finite on compact sets) $F$-invariant nonnegative Borel measures on $\partial^{2} F$. The length $L(\eta)$ of a current $\eta$ is the measure $\eta(B)$ of the base-ball $B$ of $\partial^{2} F$.

The length of a current $\eta$ is explicitly given by

$$
L(\eta)=\sum_{x \in A} \eta(\operatorname{Cyl}([1, x])) .
$$

Definition 3.3 (Frequency measures). The space of frequency measures is the set of finite-mass $T$-invariant non-negative Borel measures on $\partial F$ (where $T$-invariant means that $T_{*} \mu=\mu$ ). The total mass of a measure $\mu$ will be denoted by $\|\mu\|$.

The unitary ball of the frequency measures, that is to say, the set of probability $T$-invariant measures on $\partial F$, is sometimes called the frequency space of $F$ in the literature (see Kap05). 
We will use the letter $\eta$ primarily to denote a current, and the letter $\mu$ to denote a frequency measure. We refer the reader to Appendix A for some basic facts about currents and measures.

If $\eta$ is a geodesic current, and $x, y \in F$, by $F$-invariance, the value $\eta(\operatorname{Cyl}([x, y]))$ depends only on the label $x^{-1} y \in F$. The $F$-invariance of currents plays the role of $T$-invariance for frequency measures. With this in mind, we can construct an isomorphism between the space of geodesic currents and the space of frequency measures as follows:

$$
\eta \leftrightarrow \mu \quad \text { if and only if } \quad \eta(\operatorname{Cyl}([x, y]))=\mu\left(\operatorname{Cyl}\left(x^{-1} y\right)\right) .
$$

More precisely, one can prove (see also Kap05, Kap06a):

Theorem 3.4. The map $\alpha$ from the space of frequency measures to the space of geodesic currents, defined by

$$
\alpha(\mu)(C y l([x, y]))=\mu\left(C y l\left(x^{-1} y\right)\right)
$$

for all $x, y \in F$, is a homeomorphism with respect to the weak-* topologies. Moreover, under this correspondence, the total mass corresponds to length, that is,

$$
L(\alpha(\mu))=\|\mu\| .
$$

Proof. We only sketch the proof. The fact that $\alpha$ is well-defined and bijective can easily be proved using $F$ - and $T$-invariance. The weak-* continuity follows from Proposition A.1, while the last claim is a straightforward computation.

The identification between currents and frequency measures induces an action of $\operatorname{Aut}(F)$ on the frequency measures given by

$$
\Phi \mu=\alpha^{-1} \circ(\Phi \times \Phi)_{*} \circ \alpha \mu .
$$

Note that the action on frequency measures is not just the push-forward via $\Phi$ because the push-forward does not commute with $T$.

The fact that the length of a current corresponds to the total mass of a frequency measure will be the first ingredient of the proof of the compactness result: bounded length $\rightarrow$ bounded norm $\rightarrow$ weak compactness.

Next, we briefly discuss relations between currents and cyclically reduced words, referring the reader to Kap06b for more details.

There is a natural embedding of the space of cyclically reduced words in the space of geodesic currents (or frequency measures). Namely, if $w$ is a cyclically reduced word, we denote by $w^{+\infty}$ the ray $w w w \cdots$, by $w^{-\infty}$ the ray $w^{-1} w^{-1} w^{-1} \cdots$, and by $\gamma_{w}$ the geodesic joining $w^{-\infty}$ and $w^{+\infty}$, that is, $\gamma_{w}=\left(w^{-\infty}, w^{+\infty}\right) \in \partial^{2} F$. Then one can associate at each word $w$ the current

$$
\eta_{w}=\sum_{v \in[w]} \delta_{\gamma_{v}}
$$

where $[w]$ is the conjugacy class of $w$ in $F$ and $\delta_{\gamma_{v}}$ denotes the Dirac measure concentrated on $\gamma_{v}$. In the literature, such currents are often referred to as rational currents. Note that if $w$ is not a proper power, then $\|w\|=L\left(\eta_{w}\right)=\left\|\alpha^{-1}\left(\eta_{w}\right)\right\|$.

Definition 3.5 (Uniform current and uniform measure). The uniform current $\eta_{A}$ and the uniform frequency measure $\mu_{A}$ are defined as follows. For all $v \in F$ we set

$$
\mu_{A}(\operatorname{Cyl}(v))=\frac{1}{2 k(2 k-1)^{|v|-1}} \quad \text { and } \quad \eta_{A}=\alpha\left(\mu_{A}\right) .
$$


Note that $L\left(\eta_{A}\right)=1$ and $\left\|\mu_{A}\right\|=1$.

Remark 3.6. The uniform current is not the product $\mu_{A} \times \mu_{A}$ on $(\partial F)^{2}$. Indeed, $\eta_{A}$ is a measure on $\partial^{2} F \neq(\partial F)^{2}$, and $F$-invariance implies that neighbourhoods of the diagonal have infinite measure.

Nevertheless, the current $\eta_{A}$ is not so different from $\mu_{A} \times \mu_{A}$. Indeed, we can disintegrate $\eta_{A}$ with measures that are in the same density class as $\mu_{A}$. This means that if we cut a slice $S_{x}$ of $\partial^{2} F$ at the point $x$, namely $S_{x}=\{x\} \times\{\partial F \backslash x\}$, then there exists a continuous function $\varphi$ on $\{\partial F \backslash x\}$ such that the measure $\mu_{x}$ induced on $S_{x}$ by $\eta_{A}$ is $\varphi \cdot \mu_{A}$. A precise version of this fact is proved in Lemma A.2.

Definition 3.7 (Length of automorphisms). For any automorphism $\Phi$ of $F$ we define the length of $\Phi$ as the length of the image of the uniform current, that is,

$$
L(\Phi)=L\left(\Phi \eta_{A}\right)=\eta_{A}\left(\Phi^{-1}(B)\right) .
$$

Because of $F$-invariance of currents, $L(\Phi)$ depends only on the class $[\Phi] \in \operatorname{Out}(F)$. We set $L([\Phi])=L(\Phi)$.

Intuitively speaking, for $\Phi \in \operatorname{Aut}(\mathrm{F})$, one can think of the length of $\Phi$ as the limit, as $n$ tends to infinity, of $\left\|\Phi\left(w_{n}\right)\right\| / n$, where $w_{n}$ is a "random" cyclically reduced word in $F$ of length $n$.

\section{Proofs of the main Results}

Sketch of the proof of Theorem 1.1. Let $\left\{\Phi_{n}\right\}$ be a sequence of automorphisms of bounded length. The strategy of the proof can be summarised as follows.

Step 1. The bounded length hypothesis, together with compactness of frequency measures, implies that the currents $\Phi_{n} \eta_{A}$ have a limit $\eta_{\infty}$ (Lemma 4.1).

Step 2. The core of the proof. We study the action of $\Phi_{n}$ on $\partial^{2} F$ and on $\partial F$. The main idea is that unbounded lengths of $\Phi_{n}$ correspond to the fact that the maps $\Phi_{n}$ accumulate all the boundary on some points (the attractors). The key point is that, on the one hand, the bounded length hypothesis excludes the presence of attractors, while on the other hand, the existence of an element of $f \in F$ such that $\left\|\Phi_{n}(f)\right\|$ is unbounded implies the presence of attractors (Lemma 4.6 and Lemma 4.8). Therefore, the maps $\Phi_{n}$ keep bounded the cyclically reduced length of all elements of $F$.

Step 3. If $\Phi_{n}$ keep bounded the cyclically reduced length of all elements of $F$, then $\Phi_{n}$ has a subsequence that converges, i.e. is eventually constant (Lemma 4.10).

We now proceed to work out the details of the proof.

Lemma 4.1. Up to passing to a subsequence, the currents $\Phi_{n} \eta_{A}$ have a limit $\eta_{\infty}$ which is a geodesic current.

Proof. Since the lengths $L\left(\Phi_{n} \eta_{A}\right)$ are bounded, the total mass of the corresponding frequency measure $\Phi_{n} \mu_{A}$ is bounded. The set of non-negative measures with bounded mass on a compact space is weakly-* compact. Since $\partial F$ is compact, up to passing to subsequences, $\Phi_{n} \mu_{A}$ has a limit $\mu_{\infty}$. Such a limit is $T$-invariant because the push-forward via a continuous map is weak-* continuous. Thus, the limit is a frequency measure, which therefore corresponds to a geodesic current $\eta_{\infty}$. By continuity of the correspondence $\alpha$ between frequency measures and geodesic currents, it follows that $\Phi_{n} \eta_{A} \rightarrow \eta_{\infty}$. 
Remark 4.2. Although it is not relevant for the sequel, we note that the limit $\eta_{\infty}$ is actually a non-zero current because $L(\Phi) \geq 1$ for any $\Phi$.

Next, we proceed to study the attractors. As the next lemma shows, attractors correspond to singularities of the limit current $\eta_{\infty}$. More precisely, a Borel measure $\sigma_{1}$ is said to be absolutely continuous w.r.t. the Borel measure $\sigma_{2}$ if for any Borel set $C, \sigma_{2}(C)=0$ implies $\sigma_{1}(C)=0$. We say that a current $\eta$ has a part concentrated on a set $C$ if $\eta_{A}(C)=0$ and $\eta(C)>0$. Similarly, we say that a frequency measure $\mu$ has a part concentrated on a set $C$ if $\mu_{A}(C)=0$ and $\mu(C)>0$.

Lemma 4.3. Let $\Psi_{n}$ be a sequence of automorphisms such that the currents $\Psi_{n} \eta_{A}$ have a limit current $\eta$. Let $p, q \in \partial F$ be two distinct points. Suppose that there exist cylinders $P_{n}=\operatorname{Cyl}\left(p_{n}\right), Q_{n}=\operatorname{Cyl}\left(q_{n}\right) \subset \partial F$ such that $p_{n} \rightarrow p$ and $q_{n} \rightarrow q$, and such that there is a positive constant c for which $\eta_{A}\left(\Psi_{n}^{-1}\left(P_{n} \times Q_{n}\right)\right)>c$. Then, the current $\eta$ has a part concentrated on $(p, q)$.

Proof. Any cylinder $C \subset \partial^{2} F$ containing $(p, q)$, contains $P_{n} \times Q_{n}$ for all sufficiently large $n$. Therefore, by definition of push-forward and by hypothesis, we get (for all sufficiently large $n$ ):

$$
\Psi_{n} \eta_{A}(C)>\Psi_{n} \eta_{A}\left(P_{n} \times Q_{n}\right)=\eta_{A}\left(\Psi_{n}^{-1}\left(P_{n} \times Q_{n}\right)\right)>c .
$$

By Proposition A.1 it follows that the limit current satisfies $\eta(C) \geq c$ for any cylinder $C$ containing $(p, q)$. This implies that $\eta((p, q)) \geq c$ while $\eta_{A}((p, q))=0$, that is, $\eta$ has a part concentrated on $(p, q)$.

The following is a standard fact, which says that the only currents that can have a part concentrated on a geodesic are essentially the rational currents (recall that $\partial F$ and $\partial^{2} F$ are identified with the set of geodesic rays and of bi-infinite geodesics in the Cayley graph, respectively).

Lemma 4.4. Any frequency measure (and hence the limit $\mu_{\infty}$ ) cannot have a part concentrated on a non-periodic ray. Therefore any current (and hence the limit $\eta_{\infty}$ ) cannot have part concentrated on a non-periodic geodesic.

Proof. Any frequency measure $\mu$ has finite mass and is $T$-invariant. Hence if it has a part concentrated on a point $x$, it has a part concentrated on each point of the $T$-orbit of $x$, with the same weight. It follows that such an orbit must be finite, forcing $x$ to be periodic.

We now prove a small lemma that will be used in Lemma 4.6. We isolated this result from the proof of Lemma 4.6 because it is the only point in which we crucially use the properties of the uniform current (this will be further discussed in Section (5).

Lemma 4.5. For any $f \in F$, there exists a positive constant $c$, depending only on $f$, such that whenever two disjoint Borel-subsets $E, S$ of $\partial F$ satisfy

$$
f(\partial F \backslash E) \subset S, \quad f^{-1}(\partial F \backslash S) \subset E,
$$

then we have

$$
\eta_{A}(E \times S) \geq c\left(1-\mu_{A}(E)\right)\left(1-\mu_{A}(S)\right) .
$$

In particular, $\eta_{A}(E \times S)=0$ if and only if either $E$ or $S$ has full-measure with respect to $\mu_{A}$. 
We notice that the hypothesis that $E$ and $S$ are disjoint is redundant. We formulated the lemma in that way for reasons of compatibility with Theorem 5.3.

Proof of Lemma 4.5. The hypothesis that $E$ and $S$ are disjoint will not be used in this proof. By hypothesis we have $\mu_{A}(S) \geq \mu_{A}(f(\partial F \backslash E))$ and $\mu_{A}(E) \geq$ $\mu_{A}\left(f^{-1}(\partial F \backslash S)\right)$, and by Lemma A.4

$$
\mu_{A}(E) \geq \frac{1-\mu_{A}(S)}{(2 k-1)^{|f|}}
$$

and

$$
\mu_{A}(S) \geq \frac{1-\mu_{A}(E)}{(2 k-1)^{|f|}}
$$

from which we get

$$
\mu_{A}(E) \mu_{A}(S) \geq \frac{\left(1-\mu_{A}(E)\right)\left(1-\mu_{A}(S)\right)}{(2 k-1)^{2|f|}} .
$$

By Lemma A.3

$$
\eta_{A}(E \times S) \geq \mu_{A}(E) \mu_{A}(S) \geq \frac{\left(1-\mu_{A}(E)\right)\left(1-\mu_{A}(S)\right)}{(2 k-1)^{2|f|}},
$$

and setting $c=\frac{1}{(2 k-1)^{2|f|}}$ completes the proof.

Now the aim is to prove that the maps $\Phi_{n}$ keep bounded the lengths of all elements of $F$, so that we can apply Lemma 4.10. We do it in the following two lemmata. Namely, in Lemma 4.6 we show that if this is not the case, then there are no attractors in $\partial^{2} F$ and at most a unique attractor in $\partial F$. Lemma 4.8 will show that, up to conjugation, we can avoid the presence of a unique attractor in $\partial F$.

Lemma 4.6. Suppose that $\Phi_{n} \eta_{A}$ has bounded length (uniformly on $n$ ). Suppose that there exists an element $f \in F$ such that the cyclically reduced length $\Phi_{n}(f)$ goes to $\infty$. Then, after possibly passing to a subsequence, $\Phi_{n}$ (as maps of $\partial F$ ) pointwise converge almost everywhere to a constant. That is to say, there exists $y \in \partial F$ such that for $\mu_{A^{-}}$almost all $x \in \partial F, \Phi_{n}(x) \rightarrow y$.

Proof. By Lemma 4.1, without loss of generality we can suppose that $\Phi_{n} \eta_{A}$ has a limit $\eta_{\infty}$.

Recall that we consider $\Phi_{n}(f)$ and $\Phi_{n}\left(f^{-1}\right)$ as freely reduced words. Let $v_{n}$ be the maximal initial segment shared by $\Phi_{n}(f)$ and $\Phi_{n}\left(f^{-1}\right)$, and let $\Psi_{n}$ be the map $x \mapsto v_{n}^{-1} \Phi_{n}(x) v_{n}$. Note that $\Psi_{n} \eta_{A}=\Phi_{n} \eta_{A}$ and that $\Psi_{n}(f)$ is cyclically reduced. Up to passing to a subsequence, $\Psi_{n}(f)$ and $\Psi_{n}\left(f^{-1}\right)$ have limits, which we denote by $r_{+}$and $r_{-}$, in $\partial F$. Since $\Psi(f)$ is cyclically reduced, $r_{+} \neq r_{-}$. Note that this also implies that $r_{+}$and $r_{-}$have no common initial segment, that is, the geodesic $\left(r_{-}, r_{+}\right)$passes through 1 , the base-point of the Cayley graph.

We now show that $\Psi_{n}$, as maps of $\partial F$, converge $\mu_{A}$-almost everywhere either to $r_{-}$or to $r_{+}$.

Next, cut $\Psi_{n}(f)$ into two segments of equal length. More precisely, we set

$$
\Psi_{n}(f)=s_{n} e_{n}^{-1}
$$

where the starting segment $s_{n}$ and the ending one $e_{n}$ both have length $\left|\Psi_{n}(f)\right| / 2$ (approximated to the nearest integers). We have $s_{n} \rightarrow r_{+}$and $e_{n} \rightarrow r_{-}$. In 
particular, for large enough $n, \operatorname{Cyl}\left(e_{n}\right) \cap \operatorname{Cyl}\left(s_{n}\right)=\emptyset$, which implies $\operatorname{Cyl}\left(\left[e_{n}, s_{n}\right]\right)=$ $\operatorname{Cyl}\left(e_{n}\right) \times \operatorname{Cyl}\left(s_{n}\right)$. For large $n$, let $C_{n}$ be such a cylinder:

$$
C_{n}=\operatorname{Cyl}\left(\left[e_{n}, s_{n}\right]\right)=\operatorname{Cyl}\left(e_{n}\right) \times \operatorname{Cyl}\left(s_{n}\right)
$$

and set $E_{n}=\Psi_{n}^{-1}\left(\operatorname{Cyl}\left(e_{n}\right)\right)$ and $S_{n}=\Psi_{n}^{-1}\left(\operatorname{Cyl}\left(s_{n}\right)\right)$. Note that $E_{n} \cap S_{n}=\emptyset$.

For all $x \in \partial F$, either $\Psi_{n}(x) \in \operatorname{Cyl}\left(e_{n}\right)$ or $\Psi_{n}(f x) \in \operatorname{Cyl}\left(s_{n}\right)$, so either $x \in E_{n}$ or $f x \in S_{n}$, whence

$$
f\left(\partial F \backslash E_{n}\right) \subset S_{n} .
$$

Similarly, either $\Psi_{n}(x) \in \operatorname{Cyl}\left(s_{n}\right)$ or $\Psi_{n}\left(f^{-1} x\right) \in \operatorname{Cyl}\left(e_{n}\right)$ and

$$
f^{-1}\left(\partial F \backslash S_{n}\right) \subset E_{n} .
$$

Thus, by Lemma 4.5

$$
\eta_{A}\left(E_{n} \times S_{n}\right) \geq \frac{\left(1-\mu_{A}\left(E_{n}\right)\right)\left(1-\mu_{A}\left(E_{n}\right)\right)}{(2 k-1)^{2|f|}} .
$$

By the definition of push-forward

$$
\Psi_{n} \eta_{A}\left(C_{n}\right)=\eta_{A}\left(\Psi_{n}^{-1}\left(C_{n}\right)\right)=\eta_{A}\left(E_{n} \times S_{n}\right),
$$

and putting together these (in)equalities, we get

$$
\Psi_{n} \eta_{A}\left(C_{n}\right) \geq \frac{\left[1-\mu_{A}\left(\Psi_{n}^{-1}\left(\operatorname{Cyl}\left(e_{n}\right)\right)\right)\right]\left[1-\mu_{A}\left(\Psi_{n}^{-1}\left(\operatorname{Cyl}\left(s_{n}\right)\right)\right)\right]}{(2 k-1)^{2|f|}} .
$$

If $\Psi_{n} \eta_{A}\left(C_{n}\right) \rightarrow 0$, then either $\mu_{A}\left(\Psi_{n}^{-1}\left(\operatorname{Cyl}\left(e_{n}\right)\right)\right)$ or $\mu_{A}\left(\Psi_{n}^{-1}\left(\mathrm{Cyl}\left(s_{n}\right)\right)\right)$ converges to 1 and therefore, up to passing to subsequences, $\Psi_{n}$ converges almost everywhere either to $r_{-}$or to $r_{+}$, and we are done.

We now show that the bounded length hypothesis excludes the possibility that $\Psi_{n} \eta_{A}\left(C_{n}\right)$ stays bounded away from zero. Indeed, suppose that there exists a constant $c$ such that $\Psi_{n} \eta_{A}\left(C_{n}\right)>c$, uniformly on $n$. Then, by Lemma 4.3. $\eta_{\infty}$ has a part concentrated on the geodesic $\left(r_{-}, r_{+}\right)$, which is therefore periodic by Lemma 4.4 let $w$ be its period. We must have

$$
r_{-}=w^{-\infty} \quad \text { and } \quad r_{+}=w^{+\infty} .
$$

We may assume that the element $f$ is not a proper power. Since $\Psi_{n}$ is an automorphism of $F$, it follows that $\Psi_{n}(f)$ is not a proper power either. Therefore, by (1), for all large enough $n$, we can write $\Psi_{n}(f)$ as

$$
\Psi_{n}(f)=w^{i(n)} u_{n} w^{j(n)}
$$

where $u_{n}$ neither starts nor ends with $w$, and the exponents $i(n)$ and $j(n)$ are nonnegative and go to infinity as $n$ does. Without loss of generality, we can suppose $i(n) \leq j(n)$, so that $s_{n}$ starts with $w^{i(n)}$.

For any $0 \leq h \leq i(n)$, let $C_{n}^{h}$ be the cylinder

$$
C_{n}^{h}=\operatorname{Cyl}\left(\left[w^{-h} e_{n}, w^{-h} s_{n}\right]\right) .
$$

Note that the $C_{n}^{h}$ 's are pairwise disjoint, because $u_{n}$ neither starts nor ends with $w$. Moreover, the condition $0 \leq h \leq i(n) \leq j(n)$ implies that the geodesic segment from $w^{-h} e_{n}$ and $w^{-h} s_{n}$ passes through 1 , thus $C_{n}^{h} \subset B$ for all $0 \leq h \leq i(n)$. By $F$-invariance of currents, we have

$$
\Psi_{n} \eta_{A}\left(C_{n}^{h}\right)=\Psi_{n} \eta_{A}\left(C_{n}\right)>c
$$


uniformly on $n$. It follows that

$$
L\left(\Phi_{n}\right)=L\left(\Psi_{n}\right)=\Psi_{n} \eta_{A}(B)>c i(n)
$$

which goes to infinity as $n$ does, contradicting the bounded length hypothesis.

Thus we have proved that, after passing to a subsequence, the maps $\Psi_{n}: x \mapsto$ $v_{n}^{-1} \Phi_{n}(x) v_{n} \mu_{A^{-a l m o s t}}$ everywhere converge to a map which is constant (either to $r_{-}$or to $\left.r_{+}\right)$. Up to possibly passing to a subsequence, $v_{n}$ converges to a limit $v_{\infty}$, which is either an element of $F$ or of $\partial F$. Since the elements $v_{n}$ were the maximal initial segments shared by $\Phi_{n}(f)$ and $\Phi_{n}\left(f^{-1}\right)$, the words $v_{n} \Psi_{n}(f)$ and $v_{n} \Psi_{n}\left(f^{-1}\right)$ are freely reduced. It follows that the maps $\Phi_{n}$ converge almost everywhere, up to passing to the same subsequence, to a constant - which is either $v_{\infty}$ (if $v_{\infty}$ is an element of $\partial F$ ) or $v_{\infty} r_{-}$or $v_{\infty} r_{+}\left(\right.$if $\left.v_{\infty} \in F\right)$.

Remark 4.7. The proof of Lemma 4.6 can be adapted to prove the following more general fact. If we replace the hypothesis " $\Phi_{n} \eta_{A}$ has bounded length $\eta_{\infty}$ " with " $\frac{\Phi_{n}}{L\left(\Phi_{n}\right)} \eta_{A}$ has a limit $\eta_{\infty}$ " - which is always true up to passing to a subsequence then, we get that $\eta_{\infty}$ does not have a part concentrated on a geodesic. Indeed, if $\eta_{\infty}$ has a part concentrated on a geodesic $\gamma$, then there exists a positive constant $c$ such that for any cylinder $C$ containing $\gamma$ we have $\eta_{A}\left(\left(\Phi_{n} \times \Phi_{n}\right)^{-1}(C)\right) \sim c L\left(\Phi_{n}\right)$. As in the argument above, we must have $\gamma=\left(w^{-\infty}, w^{+\infty}\right)$ for some $w \in F$, and conjugating $\Phi_{n}$ by a suitable power of $w$, we reach a contradiction. Indeed, if $X$ denotes the set $\left(\Phi_{n} \times \Phi_{n}\right)^{-1}(C)$, then $\left(\Phi_{n} \times \Phi_{n}\right)^{-1}(w C)=\Phi_{n}^{-1}(w) X$ which is contained in the set $\left(\Phi_{n} \times \Phi_{n}\right)^{-1}(B)$, whose $\eta_{A}$-measure is $L\left(\Phi_{n}\right)$ by definition. If a geodesic belongs to $X \cap \Phi_{n}^{-1}(w) X$, then it passes through 1 , whence

$$
\eta_{A}\left(X \cap \Phi_{n}^{-1}(w) X\right) \leq 1 .
$$

Since $\eta_{A}(X) \sim c L\left(\Phi_{n}\right)$, we can conjugate by $w$ approximately at most $1 / c$ times, while if $\gamma=\left(w^{-\infty}, w^{+\infty}\right)$, then we can do that infinitely many times.

After Lemma 4.6, it remains to deal with the case where $\Phi_{n}$ converges almost everywhere to a constant. What is the behavior of such a sequence? An example can be constructed by taking a fixed $\Phi$ and conjugating with elements $v_{n}$ whose length goes to infinity. The next lemma shows that more or less this is the only possibility.

Lemma 4.8. Let $\Phi_{n}$ be a sequence of automorphisms of $F$. Then, there exists $v_{n} \in$ $F$ such that, up to possibly passing to a subsequence, the maps $x \mapsto v_{n}^{-1} \Phi_{n}(x) v_{n}$ have no subsequence converging to a constant $\mu_{A}$-almost everywhere.

Proof. The rough idea is that, via conjugations, we can force the "barycentre of $\Phi_{n} "$ to stay in a fixed compact.

For any freely reduced word $w$ of length $M$, define $B_{n}(w)$ as the set of rays $x$ such that $\Phi_{n}(x)$ starts by $w$, namely

$$
B_{n}(w)=\left\{x \in \partial F: \Phi_{n}(x) \in \operatorname{Cyl}(w)\right\}=\Phi_{n}^{-1}(\operatorname{Cyl}(w)) .
$$

Obviously $B_{n}(1)=\partial F$. Moreover, for each $n$ we have

$$
\lim _{M \rightarrow \infty} \sup _{|w|=M} \mu_{A}\left(B_{n}(w)\right)=0 .
$$

Indeed, otherwise for all $M$ there exists $w_{M} \in F$ of length $M$ such that $\mu_{A}\left(B_{n}\left(w_{M}\right)\right)$ $>c>0$. Up to subsequences, $w_{M}$ converges to a ray $R$, and $\Phi_{n}\left(B_{n}\left(w_{M}\right)\right)$ 
$=\operatorname{Cyl}\left(w_{M}\right) \rightarrow R$, contradicting the fact that $\Phi_{n}$ is a homeomorphism of $\partial F$ (in this argument $n$ is fixed).

Now, let $v_{n}$ be a freely reduced word of maximal length such that $\mu_{A}\left(B_{n}\left(v_{n}\right)\right) \geq$ $\frac{1}{2}$. Let $\widetilde{\Phi}_{n}$ be the map $x \mapsto v_{n}^{-1} \Phi(x) v_{n}$ and let

$$
\widetilde{B}_{n}(w)=\left\{x \in \partial F: \widetilde{\Phi}_{n}(w) \in \operatorname{Cyl}(w)\right\} .
$$

Let $l_{n} \in A$ be the last letter of $v_{n}$. Since $\mu_{A}\left(B_{n}\left(v_{n}\right)\right) \geq \frac{1}{2}$ we get $\mu_{A}\left(\widetilde{B}_{n}\left(l_{n}^{-1}\right)\right) \leq \frac{1}{2}$. On the other hand, for any $a \in A$, different from $l_{n}^{-1}$, maximality of the length of $v_{n}$ implies

$$
\mu_{A}\left(\widetilde{B}_{n}(a)\right) \leq \frac{1}{2}
$$

Hence, such an inequality holds for all $a \in A$. It follows that the sequence $\widetilde{\Phi}_{n}$ cannot have any subsequence converging to a constant almost everywhere.

Since conjugations do not affect the length of cyclically reduced words, Lemma 4.6 and Lemma 4.8 can be summarised as follows (recall that for $f \in F,|f|$ denotes its length, while $\|f\|$ denotes the length of the cyclically reduced word obtained from $f$ ).

Corollary 4.9. Let $\Phi_{n}$ be a sequence of automorphisms. If there is $M$ such that $L\left(\Phi_{n}\right)<M$, then for each $f \in F$ there exists $M(f)$ such that $\left\|\Phi_{n}(f)\right\|<M(f)$.

As the experts know, Corollary 4.9 implies Theorem 1.1. We include the proof of the following Lemma 4.10 for completeness.

Lemma 4.10. Let $\left\{\Phi_{n}\right\}$ be a sequence of automorphisms such that for each $f \in$ $F$ there is an $M(f)$ such that $\left\|\Phi_{n}(f)\right\|<M(f)$. Then, there exist elements $v_{n} \in F$ such that a subsequence of $\left\{v_{n}^{-1} \Phi_{n} v_{n}\right\}$ converges to an automorphism (i.e. $\left\{v_{n}^{-1} \Phi_{n} v_{n}\right\}$ has a constant subsequence).

Proof. By a diagonal argument, up to passing to a subsequence, the maps $\Phi_{n}$, as maps from $F$ to itself, pointwise converge to a map $\Phi_{\infty}$ (up to conjugation). In particular, there exists a map $\Phi_{\infty}: A \rightarrow F$ and maps $w_{n}: A \rightarrow F$ such that $\Phi_{\infty}(f)$ is cyclically reduced and, up to passing to a subsequence, for all $f \in A$ we have

$$
\Phi_{n}(f)=w_{n}(f) \Phi_{\infty}(f) w_{n}(f)^{-1} .
$$

Choose an element $a \in A$. Up to conjugation we can suppose that $w_{n}(a)=1$, that is, $\Phi_{n}$ really converges as an automorphism on the subgroup generated by $a$. Let $G \subset A$ be a maximal set of generators $g$ such that $\left|\Phi_{n}(g)\right|$ stays bounded. If $G=A$ we are done, because, up to subsequences, $\Phi_{n}$ converges on $A$, whence on $F$. Otherwise, there exists $f \in A$ such that the length of $w_{n}(f)$ goes to infinity. Since

$$
\Phi_{n}(a f)=\Phi_{\infty}(a) \Phi_{n}(f)=\Phi_{\infty}(a) w_{n}(f) \Phi_{\infty}(f) w_{n}(f)^{-1}
$$

has bounded cyclically reduced length, and since $\Phi_{\infty}(a)$ has finite length, we get that, for large enough $n, w_{n}(f)$ must start either with $\Phi_{\infty}(a)$ or with $\Phi_{\infty}(a)^{-1}$. Iterating this argument we get that $w_{n}(f)$ is the product of a power of $\Phi_{\infty}(a)$ and a word of bounded length. Thus, up to subsequences, we get

$$
w_{n}(f)=\Phi_{\infty}(a)^{m} u
$$

for some $m \in \mathbb{Z}$ with $|m| \rightarrow \infty$ as $n \rightarrow \infty$, and $u$ a finite word (which depends on $f)$. 
It follows that, up to conjugating $\Phi_{n}$ by $\Phi_{\infty}(a)^{m}$, we can suppose that $G$ has at least two elements $a, b$ and that $\Phi_{n}$ is eventually constant on the subgroup generated by $a$ and $b$. If $G \neq A$, let $f$ be as above. As in (3), we get

$$
\begin{gathered}
w_{n}(f)=\Phi_{\infty}(a)^{m} u, \\
w_{n}(f)=\Phi_{n}(b)^{l} v
\end{gathered}
$$

for some exponents $m, l$ such that $|m|,|l|$ go to infinity as $n$ does, and fixed words $u, v$ (depending on $f, a, b)$. Therefore, as $n$ goes to infinity, we get that the unoriented geodesics $\left(\Phi_{\infty}(a)^{-\infty}, \Phi_{\infty}(a)^{+\infty}\right)$ and $\left(\Phi_{n}(b)^{-\infty}, \Phi_{n}(b)^{+\infty}\right)$ coincide.

This implies that $\Phi_{n}(b)$ is cyclically reduced. In particular, we get $\Phi_{n}(b)=$ $\Phi_{\infty}(b)$, and therefore $\Phi_{\infty}$ is an automorphism on the group generated by $a$ and $b$. Moreover, the above inequalities imply that

$$
\Phi_{\infty}(b)^{\left|\Phi_{\infty}(a)\right|}=\Phi_{\infty}\left(a^{ \pm 1}\right)^{\left|\Phi_{\infty}(b)\right|},
$$

whence

$$
b^{\left|\Phi_{\infty}(a)\right|}=a^{ \pm 1\left|\Phi_{\infty}(b)\right|},
$$

which is impossible because $F$ is free. Thus $G=A$, and hence there exists a subsequence of $\left\{\Phi_{n}\right\}$ which converges.

By Lemma 4.8, up to conjugation, the sequence $\Phi_{n}$ does not sub-converge almost everywhere to the same point; by Lemma 4.6 we can apply Lemma 4.10, and the proof of Theorem 1.1 is complete.

Proof of Corollary 1.2. We have to prove that the spectrum of the length function is discrete. Suppose not, and take a sequence $\Phi_{n}$ of automorphisms such that $L\left(\Phi_{n}\right)$ has a limit $\lambda$, with $L\left(\Phi_{n}\right) \neq \lambda$ for all $n$. By Theorem 1.1 there exist elements $v_{n}$ and a subsequence $n_{i}$ such that the maps $\Psi_{n_{i}}: x \mapsto v_{n_{i}} \Phi_{n_{i}}(x) v_{n_{i}}^{-1}$ converge to an automorphism $\Psi$. Thus, the sequence $\Psi_{n_{i}}$ is eventually constant, and therefore the sequence of lengths $L\left(\Psi_{n_{i}}\right)$ is also eventually constant. But $L\left(\Psi_{n_{i}}\right)=L\left(\Phi_{n_{i}}\right)$ is therefore eventually equal to $\lambda$, a contradiction.

Proof of Theorem 1.3. This immediately follows from Corollary 1.2 and Kap06a. Proposition 5.2]. Indeed, I. Kapovich proved that for any non-simple automorphism $\Phi$ there exists a Whitehead automorphism $\tau$ such that

$$
1 \leq L(\tau \Phi)<L(\Phi)
$$

and the claim follows by an inductive argument on the length.

\section{Generalisations}

In this section we give a (partial) answer to the question: For which currents does Theorem 1.1 hold?. The idea is that length-compactness is true (for any action on metric trees and) for any current for which Lemma 4.5 holds.

Definition 5.1 ( $\eta$-length of automorphisms). Let $\eta$ be a geodesic current and let $\Phi \in \operatorname{Aut}(F)$. We define the $\eta$-length of $\Phi$ as

$$
L_{\eta}(\Phi)=L(\Phi \eta)
$$


The proof of Theorem 1.1 can be followed step by step in this new setting, obtaining:

Theorem 5.2. Let $\eta$ be a geodesic current and let $\mu$ be its corresponding frequencymeasure. Suppose there exists a constant $c>0$ and that for each $f \in F$ there is $b(f)>0$ such that for any disjoint Borel sets $E, S \subset \partial F$,

$$
\mu(f E) \geq b(f) \mu(E) \quad \text { and } \quad \eta(E \times S) \geq c \mu(E) \mu(S) .
$$

Then Theorem 1.1 holds for $\eta$. That is to say, any sequence of automorphisms $\Phi_{n} \in \operatorname{Aut}(F)$ with bounded $\eta$-length has, after possibly conjugating, a convergent subsequence (i.e. a constant subsequence).

Proof. The hypotheses on $\eta$ guarantee that Lemma 4.5 holds for $\eta$. Moreover, our assumptions imply that $\eta$ is not concentrated on a single geodesic. This implies that Lemma 4.8 can be rewritten, with the difference being that equation (2) of page 170 becomes (notation as in Lemma 4.8)

$$
\lim _{M \rightarrow \infty} \sup _{|w|=M} \mu\left(B_{n}(w)\right)<C<1
$$

for a certain constant $C$, so that we have to consider a word $v_{n}$ of maximal length such that $\mu\left(B_{n}\left(v_{n}\right)\right) \geq C$.

The proof now is exactly as in Theorem 1.1 because there are no other places in the proof of Theorem 1.1 where the specific properties of the uniform current can be used.

We now give a criterion for a current to satisfy the hypotheses of Theorem 5.2 formulated in terms of the corresponding frequency measure.

Theorem 5.3. Let $\eta$ be a geodesic current and $\mu$ be its corresponding frequency measure. Suppose that for each $a \in A$ there exist two strictly positive constants $C_{1}(a)$ and $C_{2}(a)$ such that for any $E \subset \partial F \backslash C y l\left(a^{-1}\right)$ we have

$$
C_{1}(a) \mu(E) \leq \mu(a E) \leq C_{2}(a) \mu(E) .
$$

For all freely reduced words $w=a_{0} \ldots a_{k}$, set $C_{i}(w)=C_{i}\left(a_{0}\right) \cdots C_{i}\left(a_{k}\right), i=1,2$.

If there is a constant $M$ such that

$$
\inf _{w \in F} \frac{C_{1}\left(w^{-1}\right)}{C_{2}(w)} \geq \frac{1}{M}, \quad \sup _{w \in F} C_{2}(w) \leq M,
$$

then the hypothesis of Theorem 5.2 is satisfied. Namely, there exists a constant $c>0$ and for each $f \in F$ there is $b(f)>0$ such that for any $E, S \subset \partial F$

$$
\mu(f E) \geq b(f) \mu(E) \quad \text { and } \quad \eta(E \times S) \geq c \mu(E) \mu(S) .
$$

In particular, length compactness holds for $\eta$.

Proof. By Proposition A.1, it suffices to prove the claims when $E$ and $S$ are cylinders, and, regarding the first claim, it suffices to prove it for generators. Let $E \subset \partial F$ be a cylinder and $a \in A$. Set $E_{0}=E \cap \operatorname{Cyl}\left(a^{-1}\right)$ and $E_{1}=E \backslash E_{0}$. Then

$$
\mu_{A}\left(a E_{0}\right) \geq \frac{1}{C_{2}\left(a^{-1}\right)} \mu_{A}\left(E_{0}\right), \quad \mu_{A}\left(a E_{1}\right) \geq C_{1}(a) \mu_{A}\left(E_{1}\right)
$$

and the first claim follows setting $b(a)=\inf \left\{C_{1}(A), \frac{1}{C_{2}\left(a^{-1}\right)}\right\}$.

We now prove the second claim. Let $E, S \subset \partial F$ be two disjoint cylinders. Let

$$
E=\operatorname{Cyl}\left(v_{0} v\right), \quad S=\operatorname{Cyl}\left(v_{0} w\right)
$$


with $v_{0} v, v_{0} w, v^{-1} w$ freely reduced (that is, $v_{0}$ is the maximal initial segment shared by $E$ and $S$ ). We set

$$
\begin{gathered}
E^{\prime}=\operatorname{Cyl}(v)=v_{0}^{-1} E, \quad S^{\prime}=\operatorname{Cyl}(w)=v_{0}^{-1} S, \\
E^{\prime \prime}=v^{-1} E^{\prime}, \quad S^{\prime \prime}=v^{-1} S^{\prime}=\operatorname{Cyl}\left(v^{-1} w\right) .
\end{gathered}
$$

By $F$-invariance we have

$$
\eta(E \times S)=\eta\left(E^{\prime} \times S^{\prime}\right)=\eta\left(E^{\prime \prime} \times S^{\prime \prime}\right)=\eta\left(\mathrm{Cyl}\left(\left[v^{-1} w\right]\right)\right)=\mu\left(S^{\prime \prime}\right) .
$$

Then by induction on the lengths of $v_{0}, v, w$ (and using the fact that $v_{0} v, v_{0} w$, $v^{-1} w$ are reduced) we get

$$
\begin{aligned}
& \mu\left(S^{\prime \prime}\right) \geq C \mu\left(S^{\prime \prime}\right) \mu\left(E^{\prime \prime}\right) \geq C C_{1}\left(v^{-1}\right) \mu\left(S^{\prime}\right) \frac{1}{C_{2}(v)} \mu\left(E^{\prime}\right) \\
= & c \frac{C_{1}\left(v^{-1}\right)}{C_{2}(v)} \mu\left(S^{\prime}\right) \mu\left(E^{\prime}\right) \geq C \frac{C_{1}\left(v^{-1}\right)}{C_{2}(v)} \frac{\mu(S)}{C_{2}\left(v_{0}\right)} \frac{\mu(E)}{C_{2}\left(v_{0}\right)}
\end{aligned}
$$

where $C$ is a suitable constant, and the claim follows by setting $c=C / M^{3}$.

\section{Appendix A}

Throughout the paper, we used some standard results about currents and measures. This section contains the proofs of some of these facts.

Proposition A.1. Let $m$ be a Borel measure on $\partial F$ or $\partial^{2} F$. Then, $m$ is determined by its value on cylinders. Moreover, if $\left\{m_{i}\right\}$ is a sequence of Borel measures, then $m_{i}$ converges to $m$ if and only if for all cylinders $C, m_{i}(C) \rightarrow m(C)$.

Proof. We restrict ourselves to the case where $m$ is a Borel measure on $\partial F$; an identical argument gives the $\partial^{2} F$-case. So assume $m$ is a Borel measure on $\partial F$. The characteristic function of any cylinder belongs to $\mathcal{C}_{0}(\partial F)$, and the space $V$ generated by the characteristic functions of cylinders is dense in $\mathcal{C}_{0}(\partial F)$ (the topology of $\mathcal{C}_{0}(\partial F)$ is given by uniform convergence). The first claim follows.

In addition, this implies that if $m_{i}$ converges to $m$, then for any cylinder $C$, $m_{i}(C) \rightarrow m(C)$. On the other hand, suppose that $m_{i}(C) \rightarrow m(C)$ for all cylinders $C$. Then for any $\chi \in V, \int \chi d m_{i} \rightarrow \int \chi d m$. Therefore, for any $\varphi \in \mathcal{C}_{0}(\partial F)$, if $\left\{\chi_{k}\right\} \subset V$ is a sequence converging to $\varphi$, we have

$$
\left|\int \varphi d\left(m_{i}-m\right)\right| \leq\left|\int\right| \varphi-\chi_{k}\left|d m_{i}\right|+\left|\int \chi_{k} d\left(m_{i}-m\right)\right|+\left|\int\right| \chi_{k}-\varphi|d m|
$$

where the sum of the first and the last term is bounded by $\left\|\varphi-\chi_{k}\right\|\left(\left\|m_{i}\right\|+\|m\|\right)$, which goes to zero as $k \rightarrow \infty$, uniformly on $i$. The second term goes to zero for any $k$.

Lemma A.2. For any $(x, y) \in \partial^{2} F$ let $L(x, y)$ be the length of the maximal initial segment shared by $x$ and $y$. Then we have

$$
\eta_{A}=2 k(2 k-1)^{2 L(x, y)-1} \mu_{A} \times \mu_{A} .
$$

Proof. Let $D, E \subset \partial F$ be two disjoint cylinders. Since $D$ and $E$ are disjoint, there exist $v, w \in F$ such that $D=\operatorname{Cyl}(v), E=\operatorname{Cyl}(w)$, and such that $v$ is not the initial segment of $w$ and vice versa. Let $L$ be the length of the maximal initial segment shared by $v$ and $w$ (possibly $L=0$ ). Now, let $D^{\prime}=\operatorname{Cyl}\left(v^{\prime}\right) \subset D$ and 
$E^{\prime}=\operatorname{Cyl}\left(w^{\prime}\right) \subset E$ be two cylinders. We set $\left|v^{\prime}\right|=L+a$ and $\left|w^{\prime}\right|=L+b$. We have $D^{\prime} \times E^{\prime}=\operatorname{Cyl}\left(\left[v^{\prime}, w^{\prime}\right]\right)$ and, by definition (Definition 3.5)

$$
\eta_{A}\left(\operatorname{Cyl}\left(\left[v^{\prime}, w^{\prime}\right]\right)\right)=\frac{1}{2 k(2 k-1)^{\left|\left(v^{\prime}\right)^{-1} w^{\prime}\right|-1}}=\frac{1}{2 k(2 k-1)^{a+b-1}}
$$

which can be written as

$$
\frac{2 k(2 k-1)^{2 L-1}}{\left(2 k(2 k-1)^{L+a-1}\right)\left(2 k(2 k-1)^{L+b-1}\right)}=2 k(2 k-1)^{2 L-1} \mu_{A}\left(D^{\prime}\right) \mu_{A}(E)^{\prime} .
$$

So by Proposition A.1, the restriction of $\eta_{A}$ to $D \times E$ is given by $2 k(2 k-1)^{2 L-1} \mu_{A} \times$ $\mu_{A}$. Since for each $(x, y) \in D \times E$ we have $L(x, y)=L$, we get that the restriction of $\eta_{A}$ to $D \times E$ is given by

$$
2 k(2 k-1)^{2 L(x, y)-1} \mu_{A} \times \mu_{A} .
$$

Since this holds for any $D, E$, the claim follows by Proposition A.1.

An immediate corollary of Lemma A.2 is the following.

Lemma A.3. Let $E, D \subset \partial F$ be two Borel subsets of $\mu_{A}$-positive measure. Then $\eta_{A}(E \times D) \geq \mu_{A}(E) \mu_{A}(D)$.

Proof. Just apply the Fubini-Tonelli theorem, using Lemma A.2, and the fact that $L(x, y) \geq 0$ and that $\frac{2 k}{2 k-1}>1$.

Lemma A.4. Let $E$ be a Borel subset of $\partial F$. Then for all $f \in F$

$$
\mu_{A}(f E) \geq \frac{\mu_{A}(E)}{(2 k-1)^{|f|}} .
$$

In particular, if $E$ has $\mu_{A}$-positive measure, then $f E$ has $\mu_{A}$-positive measure.

Proof. It suffices to prove the claim for $f \in A$. Let $E_{0}=E \cap \operatorname{Cyl}\left(f^{-1}\right)$ and $E_{1}=E \backslash E_{0}$. By definition of $\mu_{A}$ and Proposition A.1 we have

$$
\mu_{A}\left(f E_{0}\right)=(2 k-1) \mu_{A}\left(E_{0}\right), \quad \mu_{A}\left(f E_{1}\right)=\frac{\mu_{A}\left(E_{1}\right)}{2 k-1},
$$

and the claim follows.

\section{ACKNOWLEDGEMENT}

The author warmly thanks Pepe Burillo, Bertrand Deroin, Warren Dicks, JeanFrançois Lafont, Joan Porti, Enric Ventura, Asli Yaman and all the members of the geometric group theory research group of the UAB. The author is indebted to Armando Martino for his many comments and suggestions.

It is a pleasure to thank Ilya Kapovich for his useful observations and for having pointed out a gap in a previous version of the present work.

\section{REFERENCES}

[AFP00] Luigi Ambrosio, Nicola Fusco, and Diego Pallara. Functions of bounded variation and free discontinuity problems. Oxford Mathematical Monographs. The Clarendon Press, Oxford University Press, New York, 2000. MR.1857292(2003a:49002)

[Bon86] Francis Bonahon. Bouts des variétés hyperboliques de dimension 3. Ann. of Math. (2), 124(1):71-158, 1986. MR847953 (88c:57013)

[Coo87] Daryl Cooper. Automorphisms of free groups have finitely generated fixed point sets. J. Algebra, 111(2):453-456, 1987. MR916179 (89a:20024) 
[Fed69] Herbert Federer. Geometric measure theory. Die Grundlehren der mathematischen Wissenschaften, Band 153. Springer-Verlag, New York, Inc., New York, 1969. MR0257325 $(41: 1976)$

[Kap05] Ilya Kapovich. The frequency space of a free group. Internat. J. Alg. Comput. (special Gaeta Grigorchuk's 50's birthday issue), 15(5-6):939-969, 2005. MR2197815 (2007a:20038)

[Kap06a] Ilya Kapovich. Clusters, currents and Whitehead's algorithm. Experimental Mathematics, 16(1):67-76, 2007. MR2312978 (2008f:20043)

[Kap06b] Ilya Kapovich. Currents on free groups. In Topological and asymptotic aspects of group theory, volume 394 of Contemp. Math., pages 149-176. Amer. Math. Soc., Providence, RI, 2006. MR.2216713

[KKS05] Vadim Kaimanovich, Ilya Kapovich, and Paul Shupp. The subadditive ergodic theorem and generic stretching factors for free group automorphisms. Preprint, arXiv:math.GR/0504105, to appear in Israel J. Math., 2005.

[LS77] Roger C. Lyndon and Paul E. Shupp. Combinatorial group theory. Springer-Verlag, Berlin, 1977. Ergebnisse der Mathematik und ihrer Grenzgebiete, Band 89. MR0577064 $(58: 28182)$

[Mar95] R. Martin. Non-uniquely ergodic foliations of thin type, measured currents and automorphisms of free groups. Ph.D. thesis, University of California, Los Angeles, 1995.

[Whi36] J. H. C. Whitehead. On equivalent sets of elements in a free group. Ann. of Math. (2), 37(4):782-800, 1936. MR1503309

Departament de Matemàtiques, Edifici C, Universitat Autònoma, 08193 Bellaterra (BARCELONA) SPAIN

E-mail address: s.francaviglia@sns.it 